\title{
Neues zur Führungsaufsicht
}

\section{Hintergrund:}

Die Führungsaufsicht nach $\$ \S 68$ ff. StGB zielt auf gefährliche und/oder gefährdete Täter und hat als Maßregel der Besserung und Sicherung einen Doppelcharakter: Sie weist durch das Angebot der Hilfe und Betreuung (§ 68a Abs. 2) eine starke Nähe zur Bewährungshilfe auf, ist also grundsätzlich als Teil des Resozialisierungskonzeptes des Reformstrafrechts zu sehen - Bewährungshelferinnen und -helfer sind auch zuständig für diesen Teil der Betreuung. Andererseits ( $§$ 68a Abs. 1) unterstellt sie den Betroffenen einer Aufsichtsstelle und dient als ambulante Entsprechung bzw. Alternative zur Sicherungsverwahrung ${ }^{1}$ damit dem Schutz der Allgemeinheit.

Die Führungsaufsicht existiert in zwei Formen: kraft richterlicher Anordnung ( $\$ 68 \mathrm{I})$ und kraft Gesetzes. Letztere tritt ein, wenn eine Freiheitsstrafe von mindestens zwei Jahren (oder, bei Sexualdelikten, von mindestens einem Jahr) vollständig verbüßt worden ist oder im Gefolge einer freiheitsentziehenden Maßregel entweder bei deren Aussetzung zur Bewährung, nach der Erledigung einer Sicherungsverwahrung oder bei Aussichtslosigkeit einer Therapie im Rahmen des $\S 64$.

Die Führungsaufsicht, die durch den Richter angeordnet wird, ist dabei statistisch bedeutungslos: Die Strafverfolgungsstatistik weist für 2004 gerade noch 36 Fälle aus. Die Führungsaufsicht kraft Gesetzes kommt häufig vor: In der Begründung des aktuellen Gesetzentwurfs der Bundesregierung wird von ca. 15.000 bis 20.000 Probandinnen und Probanden der Führungsaufsicht ausgegangen (eine Gesamtstatistik fehlt), die letzten verfügbaren Daten aus der Bewährungshilfestatistik von 1999 wiesen noch 12.500 Probandinnen und

\section{Christine Morgenstern}

folge der Führungsaufsicht ihrer bisherigen Form - dort, wo Vergleichsgruppen von NichtUnterstellten zur Verfügung standen, unterschieden sich die Rückfallraten kaum; bei den ehemaligen Psychiatrie-Patienten vermuten die Autoren der Studie eher eine sorgsame Entlassungsvorbereitung und Nachsorge als Grund für die günstigen Ergebnisse.
Seit ihrer Einführung war die Führungsaufsicht Kritik ausgesetzt, die sich im Spektrum zwischen der Forderung nach Abschaffung und dem Vorwurf der Zahnlosigkeit, begleitet vom Ruf nach Verschärfung, bewegte. Die eine Position führte vor allem die Ablehnung des Instituts durch die Praxis ins Feld: Durch die Justiz, die Führungsaufsicht nicht anordnet, und insbesondere durch viele Bewährungshelferinnen und -helfer, die teilweise aus grundsätzlichen Erwägungen jegliche Überwachungselemente im Zusammenhang mit Lebenshilfen ablehn(t)en, teilweise aber einfach der Auffassung waren bzw. sind, das Institut der Führungsaufsicht sei durch eine intensivierte Bewährungshilfe zu ersetzen. Die Verfassungsmäßigkeit wurde vor allem unter den Aspekt der Verhältnismäßigkeit ${ }^{5}$ und des Verbots der Doppelbestrafung angezweifelt, vom Bundesverfassungsgericht jedoch bestätigt. ${ }^{6}$ Häufiger jedoch wurden nur einzelne Regelungen für unpraktisch oder auch rechtsstaatlich bedenklich gehalten; hier sind zum Beispiel das Nebeneinander (mitunter offenbar auch Gegeneinander) von Aufsichtsund Bewährungshilfestellen, die Strafandrohung bei einem Verstoß gegen Weisungen oder die Schaffung der Möglichkeit zur unbefristeten Führungsaufsicht für Sexualstraftäter von 1998 zu nennen.

Die Gegenposition berief sich auf Defizite der bestehenden Regelungen, etwa, dass bei Verstößen gegen Kontaktverbote keinerlei Druckmittel zur Verfügung stünden und forderte die Möglichkeit, auch auf einen Verstoß gegen die Führungsaufsichtsweisung, sich einer Therapie zu unterziehen, mit der Anordnung unbefristeter Führungsaufsicht begegnen zu können. ${ }^{7}$

geschrieben hat, ihre „effizientere praktische Handhabung“ zu ermöglichen. Bemerkenswerterweise war dieses Ziel zuvor sogar schon im Koalitionsvertrag festgeschrieben worden; hier war von „Konsequenz und Effizienz“ die Rede. Der Entwurf der Bundesregierung basiert $u$. a. auf einem Bericht des Strafrechtsausschusses der Justizministerkonferenz, der die Ergebnisse einer seit 2003 bei den entsprechenden Stellen der Länder und den Verbänden durchgeführten Umfrage zusammenstellt. Der Entwurf stellt keinen gesetzgeberischen Schnellschuss dar, sondern lässt in seiner umfangreichen und sorgsamen Begründung erkennen, dass die Anliegen der Praxis berücksichtigt werden sollten.

\section{Die Neuerungen im Überblick}

Im Folgenden werden einige der Neuerungen herausgegriffen und kommentiert.

\section{Erweiterung des Weisungs- katalogs}

Zunächst sieht der Entwurf eine Ausweitung des über $§ 145 a$ strafbewehrten Katalogs der Weisungen nach $\S 68 b$ Abs. 1 vor. Dabei wird von der Praxis auch im Sinne des Opferschutzes begrüßt, ${ }^{10}$ dass ein Kontakt- und Verkehrsverbot verhängt werden kann, über das das Opfer auf Antrag auch informiert werden muss. Ebenfalls neu ist die Weisung, keinen Alkohol oder andere Rauschmittel zu sich zu nehmen, wenn die Gefahr besteht, dass es im Gefolge des Konsums zu neuen Straftaten kommt. Bei beiden ist im Sinne des Abs. 3 der Vorschrift aber besonders darauf $\mathrm{zu}$ achten, dass es nicht zu unzumutbaren Anforderungen an die Lebensführung des Betroffenen kommt. Denn: „Jede per Weisung gegebene Verhaltensvorschrift begründet eine zusätzliche Versagensmöglichkeit". ${ }^{11}$ Die Weisungen müssen deshalb stützend wirken und dürfen nicht wegen Überforderung des Probanden geradewegs wieder in die Strafbarkeit führen. 2006 einen Gesetzentwurf zur Reform der Führungsaufsicht vorgelegt, ${ }^{9}$ der es sich auf die Fahnen 
Weisung, die eine Vorstellungspflicht in einer ärztlichen bzw. psychotherapeutischen Praxis oder einer forensischen Ambulanz beinhaltet, ist insofern sinnvoll, als die Notwendigkeit der Einrichtung forensischer Ambulanzen, die schon lange von den forensischen Psychiatern gefordert wird, anerkannt und festgeschrieben wird. ${ }^{12}$ Das gilt umso mehr für die ausdrückliche Aufnahme einer neuen Nachsorgeweisung in $\S 68$ Abs. 2 (also den "sonstigen“, nicht strafbewehrten Weisungen) und die organisatorische Einbindung der Ambulanzen in $\S 68$ a Abs. 7 StGB-E. Dass außerdem ein strafbewehrtes Vorstellungsgebot in einer Ambulanz oder Klinik neu hinzukommen soll, aus den bekannten Gründen gleichzeitig aber keine Zwangstherapien vorgesehen sind, mag seltsam erscheinen, ist aber dennoch zu befürworten. Ein Vorstellungsgebot erreicht immerhin, dass der Proband überhaupt Kontakt aufnimmt, ob eine Therapie tatsächlich zustande kommt, wird dann auch vom Geschick des Therapeuten abhängen.

\section{Heraufsetzung der Strafober- grenze bei Weisungsverstößen}

Die Erweiterung des verbindlichen Weisungskatalogs ist also überwiegend $\mathrm{zu}$ befürworten. Gleichwohl ist $\mathrm{zu}$ fragen, welchen Nutzen die Strafandrohung des $§ 145 a$ haben kann, für den der Entwurf eine Anhebung der Strafobergrenze auf drei Jahre vorsieht. Die Begründung des Entwurfs hierfür ist bei der Erörterung der spezialpräventiven Wirkung widersprüchlich: Es wird anerkannt, dass die Mitarbeiterinnen und Mitarbeiter der Führungsaufsicht in der Regel zu „Beeinflussungsstrategien im Vorfeld einer Sanktionierung greifen" inwiefern dabei eine höhere Strafandrohung Wirkung entfalten soll, ist nicht ersichtlich. Deutlicher ist dies: „In generalpräventiver Hinsicht wertet die höhere Strafobergrenze das Institut der Führungsaufsicht auch nach außen hin sichtbar auf" - es geht also wohl eher um die Befriedigung eines vermuteten Strafbedürfnisses der Bevölkerung. An der Kritik, die von jeher gegen den $\S 145$ a vorgebracht wird, ist aber festzuhalten: Fraglich ist schon, ob er als Blankettvorschrift überhaupt bestimmt genug ist. ${ }^{13}$ Selbst wenn das der Fall ist, bleiben erhebliche Zwei-fel an der Verhältnismäßigkeit, wenn letztlich ein Ungehorsam pönalisiert wird - konsequenterweise müsste das dann ja durch eine unbedingte Freiheitsstrafe geschehen. Deshalb wurde zu Recht immer wieder seine Streichung gefordert, ${ }^{14}$ Eine Strafbarkeit stellt im Übrigen auch einen Verstoß gegen Nr. 84 der Europäischen Grundsätze für gemeindebezogene Sanktionen und Maßnahmen dar. ${ }^{15}$

Die Vorschrift des $\S 145$ a gilt darüber hinaus auch nicht als effektiv: Denkbar ist zwar, dass die Vorschrift durch die Bewährungshelferinnen und -helfer als Druckmittel genutzt wird, ${ }^{16}$ tatsächlich abgeurteilt werden jedoch kaum Fälle: 2004 waren es 72 , von denen 57 mit einer Verurteilung endeten, davon wurden lediglich 27 Täter zu einer Freiheitsstrafe verurteilt, davon nur 15 unbedingt, keine Freiheitsstrafe war länger als 9 Monate. Das ist auch nachvollziehbar, weil das Antragsrecht allein bei den Aufsichtsstellen liegt, ${ }^{17}$ die versuchen dürften, die mit dem Probanden angestrebte oder erreichte Vertrauensbeziehung nicht zu belasten. Aus den Reihen der Bewährungshilfe wurde die geplante Neufassung als wirkungslos ${ }^{18}$ bzw. überzogen ${ }^{19}$ kritisiert. Im Ergebnis bleibt es dabei: Bei gröblichen oder beharrlichen Verstößen gegen Weisungen der Führungsaufsicht genügt bei den ausgesetzten Maßregelfällen die Widerrufsmöglichkeit. Bei den Vollverbüßern ist eine reine Ungehorsamsbestrafung als unverhältnismäßig ebenso abzulehnen.

\section{Schaffung eines Kriseninter- ventionsinstrumentariums}

$\mathrm{Zu}$ begrüßen ${ }^{20}$ ist die Regelung (\$ 67h des Entwurfs), wonach bei Personen, deren Maßregel nach § 63 oder $§ 64$ zur Bewährung ausgesetzt ist, auf voraussichtlich relativ kurzfristig $\mathrm{zu}$ behebende Probleme reagiert werden kann, indem für zwei Monate (zweimal

verlängerbar) der Vollzug der Unterbringung wieder in Kraft gesetzt wird. So kann auf eine Krise reagiert werden, ohne die Aussetzung insgesamt widerrufen $\mathrm{zu}$ müssen. Allerdings bleiben hier auch Fragen hinsichtlich der Praktikabilität: Die entsprechenden Kliniken müssen immer „Notfallbetten“ frei halten. Außerdem ist die Frage, wie ein schnelles Verfahren zu gewährleisten ist, wenn der Betroffene in einer Krisensituation nicht bereit ist, in die Klinik zurückzukehren.

Organisation der Führungsauf-
sicht, Erweiterung der Befug-
nisse der Führungsaufsichtstelle

In der Stellungnahme der DBH wird angemerkt, dass „...(es) insgesamt sehr darauf ankommen wird, hinreichende organisatorische Bedingungen bei den vor Ort zuständigen... Stellen der Justiz zu schaffen, damit Reformen wirksam werden können." Hier ist der Entwurf aber relativ schwach, weil er zwar für eine Vermeidung von Doppelbetreuungen sorgen will ( $§$ 68e Abs. 1 StGB-E) und die forensische Ambulanz in das System eingebunden wird (s. o.); welche Wege es aber auf dem Weg zu einem Einvernehmen grundsätzlich gibt, wie die Kommunikationswege aussehen könnten etc., wird nicht angesprochen. Immerhin wird aber klar, dass bei einer laufenden Behandlung durch die Ambulanz in die therapeutische Unabhängigkeit nicht eingegriffen werden darf, Helferkonferenzen der beste Weg wären. ${ }^{21} \mathrm{Im}$ Übrigen wird das Problem, wie die Aufgaben der Aufsicht und der Hilfe sinnvoll aufgeteilt bzw. möglicherweise durch die Schaffung einer Art „Intensivstation der Straffälligenhilfe “22 gebündelt werden könnten, nicht angesprochen. Dabei gibt es durchaus positive Beispiele aus der Praxis: Das in Darmstadt angesiedelte Modell „Konzentrierte Führungsaufsicht" fasst organisatorisch und räumlich die Leiterin der Führungsaufsichtsstelle und zwei Bewährungshelfer zu einer Einheit zusammen, die maximal 60 Probanden betreut. Das Konzept beinhaltet auch eine bessere bzw. frühzeitige Abstimmungspraxis zwischen Justizvollzugsanstalten, Staatsanwaltschaft, Strafvollstreckungskammer und der Führungsaufsichtstelle. ${ }^{23}$

Zu den Neuerungen im Verfahren gehört auch, die Führungsaufsichtsstelle $\mathrm{zu}$ bevollmächtigen, die Ausschreibung des Verurteilten anzuordnen, wenn er unbekannten Aufenthalts ist (§ 463a Abs. 1 StPO-E). Sie kann auch Vorführungsbefehle gegen solche Probanden erlassen (§ 463a Abs. 3 StPO-E), die keinen ausreichenden Kontakt halten oder der Vorstellungsweisung bei den Ambulanzen nicht nachkommen. Das erscheint grundsätzlich zweckmäßig. Rechtlich ist es allerdings bedenklich, wenn eine solche Anordnung, die unter Umständen ja mit einer zumindest kurzfristigen Freiheitsentziehung verbunden sein kann, nicht richterlich angeordnet wird. ${ }^{24}$

\section{Möglichkeit der unbefristeten Verlängerung der Führungsauf- sicht}

Bislang konnte die Führungsaufsicht längstens fünf Jahre dauern. Das soll sich nach dem Entwurf (§ 68c Abs. 3) für bestimmte Fälle ändern. Dann nämlich, wenn Gründe für die Annahme bestehen, dass die betroffene Person andernfalls alsbald wieder in ihre psychische Krankheit oder Störung zurückfallen wird und bei denjenigen, die eine längere Freiheitsstrafe (oder Maßregel) wegen der in $\S 181 b$ genannten Sexualstraftaten verbüßt haben. In beiden Fällen ist nunmehr die unbefristete Anordnung der Führungsaufsicht möglich. Sie wird von Fachleuten als nicht Erfolg versprechend kritisiert, sie führe darüber hinaus zu einer Erhöhung der Fallbelastung der Bewährungshilfe und der Gutachter. ${ }^{25}$

Maßnahmen von unbestimmter Dauer als Rechtsfolge einer Straftat sind im Hinblick auf das Bestimmtheitsgebot problematisch und können zur Unverhältnismäßigkeit führen. Die Europäischen Grundsätze für gemeindebezogene Sanktionen und Maßnahmen verboten $\operatorname{sie}^{26}$ in ihrer Fassung von 1992 aus diesen 
Gründen und angesichts der Überlegung, dass sie den Straffälligen in einem mit dem Zweck der ambulanten Sanktionen und Maßnahmen nicht vereinbaren dauerhaften Abhängigkeitsverhältnis halten können. ${ }^{27}$ Die Ausweitung der Möglichkeiten, Führungsaufsicht unbefristet zu verhängen, ist ein Schritt weg vom schuldangemessenen Sanktionenrecht und hin zu einem Präventivstrafrecht. Psychisch Kranke - auch gefährliche-Personen, die ihre Strafe verbüßt haben oder gegen die eine Maßregel nicht mehr greift, sind nicht durch das Strafrecht zu erfassen, für sie gibt es die Unterbringungsgesetze (PsychKG) der Länder.

\section{Gesamtwürdigung}

Der Gesetzentwurf macht deutlich, dass es der Bundesregierung tendenziell mehr um den Ausbau von Kontrolle und einen vermeintlichen Zugewinn an Sicherheit geht als um eine Verbesserung der Hilfsangebote. Es wird auch in der Außendarstellung des Entwurfs vorwiegend darauf hingewiesen, dass entlassene Straftäter stärker kontrolliert werden sollen. Eine Pressemitteilung der Bundesregierung zitiert die Bundesjustizministerin mit den Worten „Diese Reform soll eine straffere und effizientere Kontrolle der Lebensführung von Straftätern ermöglichen."28 Das mag bei den Bürgerinnen und Bürgern den Eindruck erwecken, einen Straftäter könne man beliebig überwachen, der Verhältnismäßigkeitsgrundsatz wird durch solche Äußerungen zumindest nicht deutlich.

Den schon lange bestehenden Forderungen nach der Abschaffung der richterlich angeordneten Führungsaufsicht nach $\S 68$ Abs. 1 wie auch des Delikts $\S$ 145a wurde - mit dem üblichen Hinweis auf die „Sicherheitsbedürfnisse der Bevölkerung “29 nicht nachgekommen, stattdessen wurde die Strafandrohung verschärft und die Möglichkeit zur Anordnung unbefristeter Führungsaufsicht geschaffen. Wurde die Führungsaufsicht einmal als ambulante Alternative zur Sicherungsverwahrung begriffen, so nehmen ihre Entwicklungen nun einen parallelen Verlauf: Beide Institute werden zu Lasten der Freiheitsgrundrechte der Betroffenen verschärft. $\mathrm{Ob}$ die Stärkung des Instituts der Führungsaufsicht geeignet ist, den Probanden vor der Sicherungsverwahrung zu bewahren, ${ }^{30}$ erscheint fraglich - wahrscheinlicher sind Net-wideningEffekte.

$\mathrm{Zu}$ kritisieren ist, dass eine echte Neukonzeption für die mit der Führungsaufsicht betrauten Stellen fehlt. Die positiv zu würdigenden Neuerungen wie die Schaffung und Einbindung forensischer Ambulanzen, die Kriseninterventionsmaßnahmen und die möglichen Verbesserungen bei Begleitung und Unterstützung insgesamt können ihre Wirkung nur entfalten, wenn dafür auch entsprechende Mittel bzw. Personal vorgesehen werden. Es mag sein, dass bei den Kosten zumindest längerfristig die Einrichtung eines Kriseninterventionsinstrumentariums durch die Vermeidung von Vollzugsaufwand wegen der Verringerung von Widerrufen kompensiert wird. Wahrscheinlicher ist aber, dass die Verschärfungen durch verlängerte oder unbefristete Führungsaufsichten und vor allem durch die nun zahlreicheren Möglichkeiten der Probanden, sich durch Weisungsverstöße Widerrufe oder eine Bestrafung einzuhandeln, insgesamt $\mathrm{zu}$ höheren Kosten führen.

Die finanziellen und organisatorischen Aspekte aber sind Ländersache, hier kann der Entwurf nur ,ein Zeichen ... setzen“,31 die Bundesjustizministerin nur an „appellieren“32. Schon aus diesem Grund ist zu fürchten, dass in der Praxis vor allem die mit den Änderungen verbundene Verschärfung und nur punktuell eine Verbesserung bei den Hilfen für Führungsaufsichtsprobanden spürbar werden.

Die Verfasserin ist wiss. Assistentin und auch Verfasserin der Rezension auf S. 156-158.

\section{Fußnoten}

2 Frehsee/Ostendorf, NK-StGB, vor $\S \S 68-68$ g Rdnr. 9

3 So z. B. in einem Fachgespräch beim rechtspolitischen Sprecher der Bundestagsfraktion von Bündnis 90/Die Grünen am 26.6.2006, http://www.jerzy-montag.de/index.php?id=562.

4 Stellungnahme der DBH zur Umfrage des BMJ im Vorfeld der Formulierung des Gesetzentwurfs an, www.dbh-online.de/stellung/dbhstellungnahme-fuehrungsaufsicht2004-03. pdf.; Streng, Strafrechtliche Sanktionen, 2002, Rdnr. 332.

5 Weigelt/Hohmann-Fricke, BewHi 3/2006, S. 216.

6 Vgl. Nachweise bei Schöch, Gutachten C für den 59. Deutschen Juristentag, „Empfehlen sich Änderungen und Ergänzungen bei den strafrechtlichen Sanktionen ohne Freiheitsentzug?, 1992, C. 109 f.

7 So klingt es in einer Stellungnahme der DBH zur Umfrage des BMJ im Vorfeld der Formulierung des Gesetzentwurfs an, www.dbh-online.de/stellung/dbh-stellungnahmefuehrungsaufsicht-2004-03.pdf.

Dünkel/Spieß BewHi 1992, S. 165.

8 BVerfGE 55, 28

9 So im BR-Entwurf vom 14.03.2005 zum Jugendstrafrecht (BRDrs.238/4).

10 Mehr zu Hintergrund und konkretem Anlass für die Gesetzesreform bei Vollbach, MSchrKrim 2006, S. $40 \mathrm{f}$.

11 BT-Drs. 16/1993.

12 DBH-Fachverband, www.dbh-online.de, Stellungnahme vom 27.9.2005; auch die Katholische Bundes-arbeitsgemeinschaft Straffälligenhilfe (KAGS), Stellungnahme vom 18.10.05, www.kags.de

13 Frehsee/Ostendorf, NK-StGB, § 68b Rdnr. 3.

14 Nedopil, „Reformvorhaben Maßregelrecht - eine Darstellung und Bewertung der geplanten Anderungen aus der Perspektive der Maßregelpraxis“, Vortrag zum Fachgespräch, vgl. Fußnote 2 .

15 Vgl. hierzu Schild NK-StGB § 145a, Rdnr. 8.

59. Deutsche Juristentag (Beschlüsse in NJW 1992, S. 3023). Dünkel/Spieß BewHi 1992, S. 166; Schöch a.a.o. C. 112

16 Morgenstern Internationale Mindeststandards für ambulante Strafen und Maßnahmen, 2002, S. 280 f.

17 DBH-Fachverband, www.dbh-online.de, Stellungnahme vom 27.9.2005

18 Nach dem Entwurf muss sie zuvor die Bewährungshilfe anhören ( $§$ $68 \mathrm{a}$ V), diese Klarstellung ist zu begrüßen.
19 So der Verteter der Bewährungshilfe beim Fachgespräch, vgl. Fußnote 2.

20 DBH-Fachverband, www.dbh-online.de, Stellungnahme vom 27.9.2005.

21 So im Grundsatz auch der DBHFachverband, www.dbh-online.de, Stellungnahme vom 27.9.2005; auch die Katholische Bundesarbeitsgemeinschaft Straffälligenhilfe (KAGS), Stellungnahme vom 18.10.05, www.kags.de und die in der Deutsche Gesellschaft für Psychiatrie, Psychotherapie und Nervenheilkunde (DGPPN) organisierten Psychiater begrüßen das Instrument, vgl. Nedopil, a.a.O.

BT-Drs. 16/1993, S. 18.

Weitere Nachweise zur Diskussion bei Frehsee/Ostendorf vor $\$ \S 68$ $68 \mathrm{~g}$ Rdnr. 17.

Sowohl das Hessische Ministerium der Justiz (PM vom 24.6.2004, www.hmdj.justiz.hessen.de) als auch die beiden Bewährungshelfer (www.bewaehrungshilfe-hessen.de, LAG aktuell 3/2003) ziehen nach Anlaufen des Projekts eine positive Zwischenbilanz.

22 Vgl. Heinhold, „Die geplanten Änderungen aus anwaltlicher Sicht unter besonderer Berücksichtigung der ausländerrechtlichen Neuerungen, Fundstelle vgl. Fußnote 2.

23 KAGS, DBH a.a.O.

24 Hierbei ist zu beachten, dass keine Trennung zwischen Strafe und Maßregel erfolgt, weil es eine solche nicht in allen Mitgliedstaaten gibt.

$25 \mathrm{Vgl}$. zur Debatte Morgenstern 2002, S. 200 ff. Eine neue Empfehlung des Europarats (Rec (2000) 22 änderte jedoch angesichts der Rechtswirklichkeit einer Reihe von europäischen Staaten den entsprechenden Grundsatz und schreibt jetzt nur noch regelmäßige Überprüfungen vor.

Die Pressemitteilung der Bundesregierung (www.bundesregierung.de) vom 5.4.2006 lautet „Entlassene Straftäter stärker kontrollieren". Dies nehmen die Medien natürlich entsprechend auf: Die Bild am Sonntag vom 19.3.2006 titelt: „Knallharte Auflagen für Sex-Verbrecher", aber auch die Agence France-Presse meldet am 18.3.2006 „Bundesregierung will für Sexualtäter Kontaktverbot $\mathrm{zu}$ Kindern einführen".

26 BT-Drs. 16/1993, S. 21 und S. 48. 27 Davon geht der Deutsche Richterbund in seiner Stellungnahme (www.drb.de) aus.

28 Entwurf S. 3.

29 Pressemitteilung vom 5.Juli 2005, www.bmj.bund.de

30 Eine ähnliche Einschätzung findet sich auch in der Stellungnahme der KAGS a.a.O. 